\title{
8 Rapidly responding to policy queries with evidence
}

\author{
Learning from Rapid Response \\ Services in Uganda
}

\author{
Ismael Kawooya, Isaac Ddumba, Edward Kayongo \\ and Rhona Mijumbi-Deve
}

\section{Summary}

The Rapid Response Service (RRS) is a knowledge translation service in Uganda that responds to a decision maker's needs for evidence with synthesised relevant evidence, contextualised and summarised in an accessible package. The RRS was set up in 2010 at the Regional East African Health Policy Initiative, Uganda node, at Makerere University, and has supported over 65 policy processes at the national and district levels. This chapter follows three cases where this evidence was used to inform policy or practice, one involving the RRS at national level, the mandatory food fortification policy, and two at district level, focusing on community distribution of misoprostol to women and reducing the turnaround time for Gene Xpert results, both in Mukono District. The evidence from the RRS was used in different ways, leading to the mandatory food fortification policy after a voluntary food fortification programme, sensitising stakeholders to implement a controversial misoprostol distribution programme to reduce postpartum haemorrhage, and to reduce the turnaround time for diagnosis of tuberculosis.

\section{Introduction}

The Rapid Response Service (RRS) is a promising knowledge translation innovation established to respond to urgent, targeted and tailored individual policy makers and institutional needs for evidence, initially in the health field. The evidence requested is synthesised, summarised and contextualised to a particular policy problem and local setting within the time needed for a policy decision to be made. The RRS at Makerere University in Uganda defines 'urgent' as a policy decision that must be taken within 28 days (Mijumbi et al., 2014). The RRS was piloted and set up in 2010 at the Regional East African Community Health Policy Initiative (REACH-PI), Uganda node, at Makerere University, College of Health Sciences, the largest and oldest academic university in Uganda. Since 2010, the service has supported over 65 health care policy processes (Mijumbi-Deve et al., 2017).

This case study uses three separate but related mini-cases to share experiences and lessons on the use of RRS and factors that enable or hinder its use. The first case relates to use at the national level, where RRS responded to policy questions 
on the national voluntary food fortification programme. The setting for the second and third cases is at the sub-national level, examining how RRS was used to support decisions by the district health team (DHT) in Mukono District.

Qualitative data collection methods, including document review and semistructured interviews, were employed for this case study. The document review included both published and unpublished documents related to the RRS food fortification programme and health services delivery at the district level. Nine key informants were purposively identified and interviewed using semistructured interviews based on their involvement in the three case studies.

\section{Understanding the context}

\section{The health sector}

The population of Uganda is estimated at 42 million as of 2018 (World Bank, 2019), with the majority residing in rural areas. Uganda is a low-income country with an estimated GDP growth of 5.3\% in 2018 (African Development Bank, 2019). Health care funding is inadequate, with total health expenditure estimated at $7.2 \%$ of GDP. The sector is characterised by a household out-ofpocket health care expenditure estimated at 41\% (WHO, 2017).

The Ugandan health care system is largely decentralised, with most primary services provided by local governments at the district or lower sub-county level, covering health care service delivery and implementation of primary health care (Bossert and Beauvais, 2002). Health care financing, planning, decision making, mobilisation of resources and coordination of services are part of the central function of the Ministry of Health (MOH) (Ministry of Environment, Water and Natural Resources, 2015).

Health care service delivery is organised through a hierarchy of administrative/ referral levels from the village health team (VHT), followed by Health Centres, which refer patients on to HC IV or district hospital level, responsible for the implementation of primary health care and supervision of the lower health facilities. The district is the next administrative level responsible for coordination, supervision and implementation of health services at the district (Ministry of Health, 2013).The regional and national referral hospitals are the higher points of health care service delivery, to which the lower health facilities eventually refer patients.

The lower level decision-making structural processes include the district health teams (DHTs) and the Health Sub-district (HSD) management team at the sub-county level, responsible for planning, organising and coordination of health services within the district and HSD, respectively. These form the extended DHT that meets once every quarter at the district and the HSD, respectively, to discuss challenges in the implementation and coordination of health programmes within the district.

Policy making can be influenced by a number of actors, such as Cabinet; other government entities, such as the Office of the Prime Minister and Ministry of Finance, Planning and Economic Development; other ministries, departments or agencies; Parliament; civil society or non-governmental organisations (e.g. Uganda National Health Consumers Organisation); the private sector; 
development partners (DPs), and public and private tertiary academic institutions involved in research.

\section{The Rapid Response Service}

The story of the RRS in Uganda begins with REACH-PI, established 15 years ago to replicate the success of the Tanzania Essential Health Interventions project (TEHIP). TEHIP, which was conducted in two districts in Tanzania in 1999, showed that the use of research evidence had significant positive effects on the implementation of policies in the communities (Kammen et al., 2006; Kasale et al., 2004). REACH-PI was therefore set up to bridge the gap between researchers and decision makers in an iterative, dynamic and interactive fashion using cutting-edge mechanisms and designs such as the piloting of the RRS in 2010 (Kammen et al., 2006). From 2010-2012, the RRS supported decision makers at the $\mathrm{MOH}$, civil society organisations, private institutions and DPs through a pilot phase, by the end of which the RRS had supported over 65 policies in two years (Mijumbi-Deve et al., 2017). Following this success, the International Development Research Council, Canada, provided funding to REACH-PI from 2015 to scale up the RRS at the national and sub-national level. There was specific interest for including the sub-national level because they were not represented in the pilot phase, despite having shown early enthusiasm for the service, and it was important to understand the factors that would enable or hinder the use of the RRS at the sub-national level (Mijumbi et al., 2014; Mijumbi-Deve et al., 2017).

RRS is demand driven and the commitment is to respond to the need for evidence to inform decisions in crisis situations (real or perceived) within 28 days. The service was set up to benefit a wide range of users in the health sector at senior to mid-levels including policy makers in government at all levels, civil society, academia, multi- and bilateral DPs and the private sector. The scope of the services is defined to include governance, delivery arrangements, health financing and health technology assessment (Mijumbi et al., 2014). Researchers hired by the RRS support policy making through searching, appraising, summarising and contextualising research evidence, and they maintain regular contact with decision makers and other stakeholders while doing so.

The service model was designed in 2010 specifically to meet the needs of the country. The need for evidence in relation to a specific policy concern or challenge is identified by a decision maker. This may include clarification of a policy problem, identifying policy options and/or implementing strategies for a policy option (Mijumbi et al., 2014). The decision maker then contacts RRS, which triggers a cascade of steps starting with clarifying the question and expressing it in an answerable format, organisational arrangements, health financing, governance, implementation strategies and health technology. Policy queries that are out of scope for the service are rejected and where possible, redirected.

After the question clarification step, the researchers search for relevant systematic reviews and appraise, contextualise and summarise the evidence in a maximum of four pages, in a jargon-free language understood by the policy maker (Mijumbi et al., 2014; Mijumbi-Deve and Sewankambo, 2017). The summary brief is then reviewed by local and external experts, often from within the RRS network or 
identified as authors in the literature cited (Mijumbi et al., 2014). Once the corrections and inputs from the review process have been responded to, the brief is submitted to the decision maker within the time agreed on between the policy maker and user.After this, the brief is used to present evidence in policy discussions, including stakeholder dialogue and debates. Recommendations emerging from stakeholder dialogue or other forums are a secondary product in the RRS.

\section{The cases}

The mandatory food fortification regulation

\section{TRANSITIONING FROM DEVELOPMENT FUNDED INITIATIVES}

Food fortification is aimed at increasing the coverage of micronutrients in the most prevalent local foods or supplements (Harvey et al., 2010; WHO Regional Office for Africa, 2013). Today, this is a priority of the Ugandan MOH Health Sector Strategic Plan (Government of Uganda, 2005; Ministry of Health, 2019) and required by law. Fortification is done for selected food products; oil, salt, maize and wheat, with micro-nutrients such as vitamins, iron, zinc and folic acid.

In 1996 at the United Nations, Uganda signed onto the global commitments to end micronutrient deficiencies. This prompted a series of initiatives, including projects financed by DPs, particularly USAID. In 2002, a National Working Group (NWG) on Food Fortification was established at the $\mathrm{MOH}$ to provide leadership in the food fortification programme. In 2004, voluntary regulations and standards for food fortification, the Food and Drugs Act (Food Fortification) Regulations were passed by Parliament and a national food fortification campaign programme was started. Only one large private industry participated in the programme, adding fortificants to oil and flour (Fiedler and Afidra, 2010).

The East Central and Southern Africa food fortification guidelines were developed in 2007, prompting all member states of East Africa to ensure standardised food fortification. Soon after, the Global Alliance for Improved Nutrition $(\mathrm{GAIN})^{1}$ provided the Ministry of Health with a grant to strengthen the voluntary food fortification programme. A multi-sectoral $\mathrm{NWG}^{2}$ was set up at the Ministry of Health with support from the director general of Health Services. In 2008 a food consumption survey supported by USAID assessed the dietary intake in the different regions in Uganda. This survey formed the benchmark for assessing the different food vehicles that could be used to deliver fortificants. The programme involved testing and purchasing of the machines and fortificants including vitamin A, zinc and iron for the private sector. This encouraged private industries to fortify oil, flour and wheat, and over $80 \%$ of industries participated and complied with food fortification regulations and standards. As such the programme was considered a success (WHO Regional Office for Africa, 2013).

The industries were ready to do it and that made it possible. This was a very expensive venture and that is where the development partner came in. We 
had to ship in most of the equipment that was used. The fortificants were very expensive.

(Respondent 7 - Non-government)

SEEKING EVIDENCE TO INFORM DIALOGUE AND THE DEVELOPMENT OF STRATEGIES FOR SUSTAINABILITY

In 2011, the grant was coming to an end and concerns emerged around the sustainability of the programme as industrial food fortification was still voluntary and the cost heavily subsidised by a grant. Since these costs would be assumed by the private industries, it was feared that they would drop out. Consequently, a representative of the DP for GAIN approached a researcher from the RRS to request evidence for the steps to ensure that the programme continued after the funding ended.

The RRS provided the following two relevant rapid response briefs:

- The first affirmed that food fortification was a proven and preferred strategy for alleviating micronutrient deficiencies because it was shown to be effective, cost effective, and achieved a wider population coverage in high quality studies.

- The second summarised evidence on how a public health (food fortification) programme can be sustained. A key message was that successful implementation and careful consideration of sustainability at the inception of the programme are essential components for sustainability.

These briefs enabled the DP to request REACH-PI to coordinate a national policy dialogue with the $\mathrm{MOH}$ and all stakeholders around the sustainability of the programme. Stakeholders from the NWG were first consulted on the sustainability of the programme. Their inputs were incorporated into a draft report that was tabled for discussion during the dialogue. With much input from the DP who met the costs for fortification, consensus was reached on the need for mandatory regulations because the food fortification programme had been voluntary.

The DP noted that there was a danger of reversing these successes if the costs for fortification are incurred by some willing private industries. They would be forced to pass on these costs to the final consumer, making their products more expensive and less competitive. A mandatory policy was therefore necessary to ensure all industry players were obliged to fortify the included foods.

\section{Community distribution of misoprostol to women in Mukono district}

\section{PILOTING A CONTROVERSIAL HEALTH STRATEGY}

In 2009, the reported maternal mortality rate in Uganda was high, averaging 438 per 100,000 live births (Uganda Bureau of Statistics (UBOS) and ICF International Inc, 2012). It was estimated that almost one in four maternal deaths was due to postpartum haemorrhage (PPH). Pregnant women were 
delivering at home without medical support because of the inadequacies at health facilities such as shortage of health workers, no refrigerators and stock outs of uterotonics such as oxytocin.

During this period, the WHO issued a recommendation that misoprostol be used in the third stage of labour where superior uterotonics such as oxytocin were not readily available (WHO, 2009). An opinion piece in Lancet 2011, advocated for community distribution of misoprostol as a way of ending morbidity and mortality due to PPH (Potts et al., 2010). A number of DPs including the Maverick Collective's Population Service Initiative (PSI) supported this recommendation and several low-income countries began providing misoprostol in peripheral health facilities.

The Programme for Accessible Health Communication and Education (PACE) Uganda, a local non-governmental organisation, conducted an initial pilot in Mubende District in 2012 that proved the feasibility of distributing misoprostol to pregnant mothers during the last trimester. With funding from the PSI, PACE Uganda advocated for community distribution of misoprostol to the $\mathrm{MOH}$ and requested to test its feasibility in five selected districts. An implementation strategy was designed by PACE, experts at the $\mathrm{MOH}$ and an independent expert from Makerere University. A key focus was to ensure restricted supply, and a robust communication strategy to ensure the proper use of misoprostol. Once approved by the MOH, PACE Uganda engaged the DHTs in the selected districts.

\section{SEEKING EVIDENCE TO MINIMISE RISK}

In rolling out this strategy, PACE Uganda approached the DHT at Mukono district for their input and approval for a pilot programmatic study involving distributing misoprostol using the existing emergency kits for pregnant mothers, known as a 'mama kit'. Interviewees noted a number of problems, however.

The most common indication for misoprostol is abortion, which made it controversial in the community. Its misuse for abortions would create a perceived conflict of interest of the Implementation Partner (IP), PACE Uganda, whose projects focused on sexual reproductive health, particularly family planning. In addition, misoprostol is only available with a doctor's prescription and through a pharmacist. Instead, the pilot study was proposing distribution through VHTs, who had minimal academic qualifications.

Misoprostol had conflicting issues. We have a partner who has been working in family planning, that is PACE then comes out with a different project which was using VHTs to distribute 'mama-kit' which contained misoprostol and that was not a policy. So we thought it prudent that it not being a policy, there would be issues which Mukono District might answer. (Respondent 1 - Government)

Before accepting the pilot programme, the district needed surety against misuse of the drug and evidence of due diligence on the effects of community 
distribution of misoprostol. The DHT requested the RRS for support to gather evidence around the optimal distribution mechanisms of misoprostol to inform a meeting between the DHT and PACE Uganda about the way forward.

My District Health Officer (DHO) is proactive and he fears (being) incriminated. So he said, [mentions name], you better ask [RRS] and see what they tell us because if they give misoprostol and they get issues it will be me who gets to answer which means that the DHO is fully answerable to whatever they [IP] do [sic].

(Respondent 2 - Government)

The research question was clarified thus: 'How can distribution of misoprostol to pregnant women for the prevention of PPH be optimised?' The brief described three models for the distribution of misoprostol, depending on who administers it. They also emphasised that evidence from the studies showed that providing misoprostol to pregnant women did not reduce health facility deliveries.

\section{Reducing the turnaround time for Gene Xpert results for TB in Mukono district}

In 2012, Uganda started using the Gene Xpert MTB/RIF ${ }^{3}$ in selected health facilities to improve the diagnosis and burden of tuberculosis by improving the case detection rate (Hanrahan et al., 2016). However, because of the prohibitive costs of purchasing and maintaining the machines and cartridges, a few selected facilities with high patient volume centres were made central referral facilities for more than one peripheral facility for Gene Xpert MTB/RIF. Motorcycle riders followed predetermined schedules and routes to transport sputum specimens to and from central facilities.

Respondents noted that this system caused delays in returning results to patients and led to dropouts.

Because we have hub riders the problem by then was that the hub riders were not delivering the results in time. We had cases where patients were waiting for results for close to two months and with the rapid response team we were guided. We increased the number of hub riders.

(Respondent 8 - Government)

DHT had quality improvement meetings in 2015 to identify and improve efficiencies, for example, increasing the number of riders and trips to facilities. However, this was not successful in addressing challenges.

They were trying to increase hub riders then too, they wanted to get someone who can sit down and sort the results. The results were being mistakenly taken to other facilities and yet there were still issues. Even though you get someone to sort results still the[re] would be errors because they are 
human. And also they had improvised that as we wait for the Gene Xpert $\mathrm{MTB} / \mathrm{RIF}$ to do $\mathrm{ZN}{ }^{4}$

(Respondent 1 - Government)

The Mukono DHT approached the RRS for relevant evidence for a brainstorming meeting with the IP about improving the turnaround time for Gene Xpert MTB/Rif. The question was: 'How can the sputum specimen referral system be strengthened to reduce the turnaround time in Mukono district?' The brief to the policy maker summarised relevant evidence, which was scarce at the time. However, based on the experiences of the early infant diagnosis specimen referral system in HIV, three options were suggested: considering adopting innovative technologies such as SMS/GPRS printers, using VHTs to link patients to the sputum specimen referral, and conducting a systems diagnosis for a local cause.

\section{Using the evidence}

It is not always possible to isolate a linear relationship between the generation and use of evidence obtained from the RRS, including its conceptual, symbolic or instrumental use. Conceptual use refers to using evidence to elucidate a policy during discussions; symbolic use refers to using evidence to legitimise or support a predetermined position; and instrumental use refers to direct and specific use in the decision-making process (Amara et al., 2004).

\section{Conceptual use}

Evidence from the RRS is often used by the decision maker to stimulate debate and clarify issues and evidence in policy-making forums. Evidence relating to the national food fortification programme focused on ensuring sustainability of the programme and was taken up in the national policy dialogue. The stakeholders from the NWG on Food Fortification discussed the challenges of ensuring sustainability with little or no ownership from the $\mathrm{MOH}$, and the importance of having adequate resources. At first, it was feared that the mandatory programme would be unsustainable for participating private industries because the costs of fortification would make their products more expensive and less competitive than those of industries not fortifying. Some industries who had been left out of earlier discussions but who were necessary for the success of the programme were brought into the National Policy dialogue discussions. These included the Ministries of Justice and Trade.

This had to be done properly to get the industry on board. It was important for them to be part of the process from the start. I have to acknowledge the efforts of private sector import in the fortification.

(Respondent 7 - Non-government) 
An example of conceptual use at district level was that the evidence provided to the district clarified initial perspectives about the possible abuse of misoprostol and a mistrust in how distribution was to be achieved. The evidence confirmed that there were no reported cases of abortions among pregnant women provided with misoprostol at over 12 weeks of gestation, and that the distribution actually increased the number of health facility deliveries. The district also used evidence of the successful distribution of misoprostol to sensitise the community and health care workers.

I was surprised actually it had worked. In your evidence, they had done a trial in Mubende. I did not know that. We did it in our own context. We modified it to suit our contexts.

(Respondent 1 - Government)

\section{Symbolic use}

Evidence from RRS can be used to support a pre-determined position of the policy maker. For example, the district leadership articulated concerns about the distribution of misoprostol in the community, anticipating possible repercussions if it affected any woman adversely. The synthesised evidence clarified a number of perceptions about any possible adverse effects.

\section{Instrumental use}

Evidence can be used directly in the formulation of policy options and/or implementation strategies. For example, the brief provided by the RRS to the district health leadership identified strategies for increasing the efficiencies and turnaround time for Gene Xpert MTB/RIF. Some of the suggestions were implemented, for example, the use of GPRS printers for all hub points and mobile phones.

The issue was delay. Then when that was done, they had to connect CPHL servers to Mukono servers. So as soon as they are done, they are relayed as they are done. The other thing was they would send text messages to those facilities which were far that these were results for patients a, b, c and $d$. Then others they would print. Then in a space of three days, the patient would get treatment there and then.

(Respondent 1 - Government)

\section{Understanding the factors that enabled and hindered use of evidence}

\section{Demand for evidence from RRS}

Important drivers for the demand for evidence from the RRS included the presence of champions and need. RRS is demand driven and responds to needs 
identified by the decision makers. All three examples cited earlier were difficult or controversial and therefore unlikely to have been resolved without evidence to inform and bridge the different views.

Another driver of demand is the need to justify funding to, for example, DPs. The users often want use evidence to improve their justification for funding.

There was credible information that had to be trusted and this also motivated the DPs to continue funding. Actually, the food fortification programme is still going on.

(Respondent 7 - Non-government)

\section{Use interventions that triggered change to enable use}

The briefs provided by the RRS do not in themselves trigger use of the evidence they contain. A number of interventions are often used concurrently to strengthen uptake and eventual use. These are illustrated in the examples in the following subsections.

\section{Visibility}

The team at Makerere University invests in ensuring that decision makers are informed of the existence, value added and importance of RRS. They do so through regular formal and informal interaction with networks at the districts and the $\mathrm{MOH}$ in trainings, meetings, etc. For example, in the food fortification programme, the policy maker heard about the RRS from an acquaintance who had received an RRS brief. She then requested evidence on the sustainability of the food fortification programme. In another example, in Mukono the RRS sensitised leaders about the RRS in 2016 as it piloted its sub-national phase.

The team also engages in advocacy for the research product using social marketing and so forth. Research is also carried out to understand the target group and how best to communicate and package messages, ensuring that communication, awareness raising, and advocacy are effective.

These efforts created awareness about and positive attitudes towards RRS, enabling use of evidence in decision making.

\section{Decision-making cultures}

The interviewees noted more demand for evidence in units where decisions are taken through comprehensive and inclusive consultations, which then enables evidence use. At district level the review meetings spurred a demand for evidence.

Evidence was existing in the district but in a rudimentary way. We have got a district quality assurance committee, so they used to do analysis ... and 
they come up with strategies, but those strategies were not informed by evidence but still it was there. So, when evidence comes in it just bridges.

(Respondent 1 - Government)

\section{Accessibility}

RRS provides evidence contextualised to the policy maker's setting, appraised and summarised in simple language and an accessible format to facilitate decision making. Relevance and accessibility ensure understanding of the evidence and how it relates to policy questions and needs.

\section{Dialogue and interaction}

The RRS process includes dialogue and interaction between researchers, decision makers and members of the public with a stake in the particular policy question. Respondents also noted that evidence use is increased when those who generate and use the information are involved in the evidence-informed decision-making process. For example, in the cases at the district level in this chapter, the assistant DHO attended training on the RRS for district officials and maintained contact with the team about different policy concerns he needed support with before the evidence request was put forward to RRS.

Regular dialogue and interaction allow for the building of relationships and trust between decision makers, stakeholders and researchers as well as an understanding of one another's realities and perspectives, all of which can be central to enabling evidence use.

\section{Demand-driven approaches}

RRS responds to queries and requests raised by decision makers. The specific evidence is requested to address a specific policy concern and through an iterative engagement process between the decision maker and researcher, the questions are clarified and defined. This ensures ownership of the evidence generated and increases the probability of use.

\section{Credible processes}

The generation of evidence by known and trusted experts in a particular area enables trust in the evidence, increasing the likelihood of use. The decision makers view the systematic and transparent processes and neutrality of the RRS as important for the use of evidence.

The game changer was when we engaged [mentions name] who had done his studies around that. He is an authority in Uganda about maternal and child health. So, he gave a lot of input on how we would manage the mothers and how we would exclude, for example, those with complicated 
pregnancies. We would exclude them because that would increase their risk for PPH.

(Respondent 5 - Non-government)

Another strategy to ensure credibility was submitting the briefs to trusted, knowledgeable leadership in the particular area, who then present it to stakeholders.

\section{Changes in capability, motivation and opportunity}

The RRS provides training to decision makers to sensitise them about the value for evidence-informed decision making, and how to find and appraise the evidence. Through working with researchers, the decision makers develop the ability to articulate their needs for evidence as they become more aware of the question clarification process and are able to define their policy queries.

The RRS promotes evidence use by responding promptly to decision makers' needs. This motivates them to seek evidence and increases their confidence during discussions.

\section{Barriers and enablers to use}

Evidence is generated and use interventions employed in a wider context that can influence actual use of evidence positively or negatively. Factors identified in the three cases enabling and hindering use of evidence generated from RRS are discussed in further detail in the following subsections.

\section{Macro-context}

\section{LEVELS OF PRIORITY}

The profile of a particular policy matter can be raised by external influences, within the region or more globally. For example, the East, Central and Southern Africa Community was influential in ensuring that Uganda undertake a food fortification programme according to the set standards, making it relevant to consider evidence for the sustainability of the food fortification programme. The pilot of the community distribution of misoprostol followed a recommendation from the WHO in 2009 to justify its implementation.

It emerged that evidence for the policy of a specific programme is easier to use if it has been identified as a priority within the country. For example, the food fortification programme was mentioned both in the Health Sector Strategic Plan and the National Development Plan, making the evidence for the sustainability of the programme relevant to the ministry's objectives, thus giving the RRS the ability to convene a national policy dialogue ( $\mathrm{MOH}, 2019)$. 
Respondents noted that decentralisation enables officials at the lower level of the health system to seek evidence for a policy concern by giving them autonomy to make decisions. A stakeholder noted that being able to make an impactful decision allows these officials to consider evidence that can affect a public health programme within their jurisdiction.

(Respondent 1 - Government)

\section{POLITICAL INFLUENCE}

Respondents noted that politicians at any level can influence a community's opinion, thus thwarting a course of action. In such situations, the technical leadership seeks evidence as an insurance policy. This was highlighted in the case for the distribution of misoprostol where any adverse event would make it hard to argue for misoprostol with the politicians if there was no evidence.

We work with politicians. If you make an issue and the mother dies, even though you have a Ministry of Health letter, the DHO will call on 'kanzindalo (megaphone)' to explain why the mother died and the DHO sent people to kill. They were like I would rather have evidence to back me up if it goes beyond my limit of control.

(Respondent 2 - Government)

Where there is political support and/or demand for evidence from politicians a respondent noted that:

Then also you can [also] think about the political support was also key, because Mukono has most of the time been working with our political leaders [sic] and they have helped us a lot especially in mobilisation, supporting some of the things that are supposed to be approved by the local council.

(Respondent 2 - Government)

\section{Institutional context}

\section{RELATIONSHIPS AND TRUST}

Interview respondents discussed the impact of relationships and levels of trust between the knowledge broker and the district leadership on positive perspectives and responses to the evidence. 'Having good working relationship on social grounds can make you really make a positive decision.... but if you are not on good terms, a positive decision becomes a problem' (Respondent 8 - Government). 
Organisational cultures and capabilities

\section{DECISION-MAKING RESPONSIBILITIES}

Interviewees noted that how individuals at different levels perceive their responsibilities can affect their demand for evidence and its eventual use. For example, where district officials saw themselves as implementers rather than decision makers, they did not engage with the evidence (Respondent 1 - Government). They indicated that once a department or institution is empowered to take policy decisions, it is more likely to consider evidence in making them. At local government level, the district health office is empowered to take decisions related to health and only has to inform the Chief Administrative Officer, who is the overall accounting officer for the district.

In addition, stakeholders also noted the importance of empowering individuals, especially at junior levels, to undertake policy decisions and therefore seek evidence for those decisions.

\section{STRUCTURES AND PROCESSES}

Stakeholders noted that evidence is more likely to be considered in a department that demands accountability. Respondents noted that health District League Tables, which are published and presented at annual gatherings at the Joint Review Missions, are an accountability tool that encourages policy makers to consider evidence to improve their performance.

Stakeholders also reported that the way the system is structured to report its findings affects how evidence is used. Health care policy makers are required by the system to report on the performance of specific indicators, particularly quantitative ones. They therefore consider evidence to improve quantitative measurements but not the quality of processes in implementation.

Feedback mechanisms were also identified as being important. Respondents gave the example of Mukono District that uses feedback as a form of quality assurance for the RRS brief once submitted to the district. This feedback mechanism encourages discussion on the evidence provided.

Interviewees noted that having mechanisms for generating data facilitates the demand for evidence and subsequently a culture where evidence is sought for a number of policy concerns. For example, at the district level, the Health Management Information System is used by biostatisticians to inform decisions at the specific level. In situations like Mukono District, where policy makers have been actively using this system to understand their performance and seek strategies, they are often eager to seek evidence for better strategies to improve their performance.

\section{INCENTIVES AND DISINCENTIVES}

Policy makers interviewed noted that they might consider the use of evidence if there were personal incentives, such as recognition of personal achievement of positive outputs from the use of evidence, and where policy makers are 
open-minded towards new initiatives. For example, a policy maker at Mukono District reported the uptake of evidence from the RRS was possible because the leadership was open-minded.

On the other hand, stakeholders interviewed noted that certain policy makers view the use of evidence as an extra task and therefore do not seek it. They would rather repeat plans from previous years because this is less demanding.

Actually, most of the districts use evidence, if you are to ask districts for the last five years. Give us your annual work plan. They are all the same. But do you think things change? No, because the [official] only does it because he has to. He does not put an extra effort to see what does not work in the previous year, what can we change?

(Respondent 1 - Government)

Respondents noted that previous results from using evidence affect future demand or use. Once the evidence has been shown to solve prior challenges, the policy makers are more inclined to evidence to solve challenges.

\section{CHAMPIONS AND LEADERS}

Stakeholders noted that champions are important for the consideration and uptake of evidence. Evidence champions are individuals in positions of influence who favour evidence use in policy and implement calculated approaches to increase the use of evidence in decisions. Their influence has been shown in studies on the factors affecting uptake of evidence-informed decision making (Basaza et al., 2018). For example, in the national food fortification programme, a stakeholder noted the importance of having top management as champions at the MOH (Respondent 7 - Non-government).

Champions can also be politicians. Instances were reported where politicians demanded accountability from technocrats. This, in turn, pushed the demand for evidence to improve the performance of government programmes. As one interviewee noted:

And [also] having political heads who are also demanding. If you have political heads who are making noise in the community, then they ask you what new thing you are doing, and you do not show anything - man, it is better you just leave.

(Respondent 1 - Government)

\section{Capacities}

SKILLS AND KNOWLEDGE FOR EVIDENCE USE

Respondents noted that certain skills are important for the consideration and use of evidence. For example, a stakeholder pointed out that leaders need the skills to search for evidence using a computer, the lack of which might limit 
their access and use of evidence 'someone who can sit on the internet, navigate the options and look for knowledge' (Respondent 1 - Government).

Interviewees also said that knowing how to generate and analyse data and search for evidence is important for evidence-informed decision making because it stimulates demand for evidence. A policy maker noted that many policy makers do not use evidence because they do not know where to find it, and therefore sensitising them about RRS has been very important.

RESOURCES

Stakeholders reported that a department needs internet and computer services to access evidence and services from the RRS, and that it enables them to search for evidence after training (Respondent 1 - Government).

\section{Reflecting and learning from the use of evidence}

\section{How context and intervention influenced the use of evidence}

The use of evidence described in this study takes place within the wider context where (1) services are largely decentralised to local governments; (2) decision making takes place at different levels; and (3) DPs play a significant role in influencing policy and driving the use of evidence. Mechanisms used to ensure the use of the service provided by RRS (i.e. evidence) include building understanding and trust in RRS through capacity-building and awarenessraising interventions. Importance is also given to building trusted relationships between decision makers, stakeholders and researchers through regular dialogue and intervention. Credibility and ownership are ensured by engaging with individuals known and trusted, the use of a tried and tested methodology, responding directly to demands, and working closely with the decision makers to ensure that demand is clearly articulated. The evidence itself is then presented in a manner that is relevant and accessible to the decision maker and is often accompanied by dialogue and discussion to allow all stakeholders to engage with it.

In the three cases described in this chapter, these interventions have successfully led to direct instrumental use of evidence. Other cases have elements of symbolic use, where evidence simply validates a decision maker's existing position.

\section{Lessons for the health sector}

The health sector in Uganda has had a number of initiatives supporting the use of evidence at $\mathrm{MOH}$. However, challenges to evidence-informed decision making becoming part of the policy processes in the health system include access to evidence, interest of stakeholders and availability of resources. Knowledge brokers such as RRS increase policy makers' access to evidence. However, 
it is important that the knowledge brokers have sufficient understanding of context to present evidence and recommendations that are socially and culturally appropriate, and thereby increase the acceptance of evidence use in the decision-making processes.

Its adoption is at a nascent stage in districts, with the RRS being one of the few initiatives attempting to support the work of decision makers at the district level. It is clear that increasing access to and availability of evidence serves the district health officers' overwhelming demand for evidence and therefore increases the possibility of evidence-informed decision making at the districts. These efforts are hampered by the limited visibility and awareness of the services RRS provides. There is a need to ensure the visibility and relevance of the service, thereby raising the awareness of the intended users and promoting its continued application, especially at the national level.

\section{Lessons for the country}

The experiences of RRS demonstrate that research evidence can promote the efficient and effective use of resources and minimise wastage. However, the use of evidence in health policy making in Uganda and beyond is still suboptimal despite efforts to bridge the research-to-action gap. It is important to reflect and learn from experience, as small but significant changes can make a huge difference. For example, at the district level, the quarterly review meetings could add a requirement that the evidence needs to be referred to prior to the adoption of an implementation strategy.

\section{A final thought}

An important lesson from the three case studies is that evidence needs an advocate to attract the attention of the decision maker. Investments are required upfront by the evidence advocates to strengthen the readiness of evidence users to demand and use the evidence, for example, by creating awareness of the existence and value of the evidence and building skills necessary to interrogate and apply evidence. Throughout the process, parallel actions can make a significant difference. These can include advocacy around the topic and the research process, building of relations and, perhaps most importantly, establishing trust in the process and the outcomes.

\section{Notes}

1 Global Alliance for Improved Nutrition is a UN Swiss-based foundation set up to tackle malnutrition globally.

2 Comprising MOH, Department of Food Science and Technology, Makerere University, Ministry of Agriculture, Animal Industries and Fisheries, National Agriculture Research Organization, Uganda Bureau of Statistics (UBOS), the Uganda National Bureau of Standards (UNBS), Ministry of Trade, the National Drug Authority (NDA), Ministry of Justice, and the Food Biosciences Research Centre (WHO Regional Office for Africa, 
2013). Food fortification is a multisectoral response to micronutrient deficiency in women and children in Uganda. Brazzavile, Republic of Congo: Regional Office for Africa.

3 TB/RIF refers to Mycobacterium tuberculosis complex/Resistance to rifampicin.

$4 \mathrm{ZN}$ is a test for tuberculosis, also known as the Ziehl-Neelsen stain.

\section{References}

African Development Bank. 2019. Uganda economic outlook [WWW Document]. African Development Bank - Build. Today Better African Tomorrow. Retrieved 15 August 2019, from www.afdb.org/en/countries/east-africa/uganda/uganda-economic-outlook.

Amara, N., Ouimet, M. and Landry, Ré. 2004. New evidence on instrumental, conceptual, and symbolic utilization of university research in government agencies. Science Communication, 26, 75-106. https://doi.org/10.1177/1075547004267491

Basaza, R., Kinegyere, A., Mutatina, B. and Sewankambo, N. 2018. National framework for the sustainability of health knowledge translation initiatives in Uganda. International Journal of Technology Assessment in Health Care, 34, 120-128. https://doi.org/10.1017/ s0266462317004482

Bossert, T.J. and Beauvais, J.C. 2002. Decentralization of health systems in Ghana, Zambia, Uganda and the Philippines: A comparative analysis of decision space. Health Policy Plan, 17, 14-31. https://doi.org/10.1093/heapol/17.1.14

Fiedler,J.L. and Afidra, R. 2010.Vitamin A fortification in Uganda: Comparing the feasibility, coverage, costs, and cost-effectiveness of fortifying vegetable oil and sugar. Food and Nutrition Bulletin, 31, 193-205. https://doi.org/10.1177/156482651003100202

Government of Uganda. 2005. Food and drugs (Food Fortification) regulations, 2005. Government of Uganda, Kampala, Uganda.

Hanrahan, C.F., Haguma, P., Ochom, E., Kinera, I., Cobelens, F., Cattamanchi, A., Davis, L., Katamba, A. and Dowdy, D. 2016. Implementation of Xpert MTB/RIF in Uganda: Missed opportunities to improve diagnosis of tuberculosis. Open Forum Infectious Diseases, 3, ofw068-ofw068. https://doi.org/10.1093/ofid/ofw068

Harvey, P., Rambeloson, Z. and Dary, O. 2010. The 2008 Uganda food consumption survey: Determining the dietary patterns of Ugandan women and children. Kampala: USAID.

Kammen, J. van, Savigny, D. de and Sewankambo, N. 2006. Using knowledge brokering to promote evidence-based-policy making: The need for support structures. Bulletin of the World Health Organization, 84, 608-612.

Kasale, H., Mbuya, C. and Lobulu, W. 2004. Case study confirms TEHIP's lead role in better health. TEHIP News.

Mijumbi, R.M., Oxman, A.D., Panisset, U. and Sewankambo, N.K. 2014. Feasibility of a rapid response mechanism to meet policymakers' urgent needs for research evidence about health systems in a low income country: A case study. Implementation Science, 9, 114. https://doi.org/10.1186/s13012-014-0114-z

Mijumbi-Deve, R., Rosenbaum, S.E., Oxman, A.D., Lavis, J.N. and Sewankambo, N.K. 2017. Policymaker experiences with rapid response briefs to address health-system and technology questions in Uganda. Health Research Policy and Systems, 15, 37. https://doi. org/10.1186/s12961-017-0200-1

Mijumbi-Deve, R. and Sewankambo, N.K. 2017. A process evaluation to assess contextual factors associated with the uptake of a rapid response service to support health systems' decision-making in Uganda. International Journal of Health Policy Management, 6, 561-571. https://doi.org/10.15171/ijhpm.2017.04 
Ministry of Health. 2013. Guidelines for governance and management structures. Kampala: Government of Uganda.

Ministry of Health. 2019. Nutrition [WWW Document]. Retrieved 28 March 2019, from https://health.go.ug/departments/nutrition.

Potts, M., Prata, N. and Sahin-Hodoglugil, N.N. 2010. Maternal mortality: One death every 7 min. Lancet, 375, 1762-1763. https://doi.org/10.1016/s0140-6736(10)60750-7

Uganda Bureau of Statistics (UBOS), ICF International Inc. 2012. Uganda demographic and health survey 2011. Kampala, Uganda: UBOS, Calverton, MD: ICF International Inc.

WHO. 2009. WHO guidelines for the management of postpartum haemorrhage and retained placenta (No. 978924159851 4). Geneva, Switzerland:WHO.

WHO. 2017. Primary health care systems (PRIMASYS): Case study from Uganda, abridged version. Geneva, Switzerland:WHO.

WHO Regional Office for Africa. 2013. Food fortification: A multisectoral response to micronutrient deficiency in women and children in Uganda. Regional office for Africa, Brazzavile, Republic of Congo.

World Bank. 2019. The World Bank in Uganda [WWW Document]. 